\title{
Influence of Nuclear Track Potentials in Insulators on the Emission of Target Auger Electrons
}

\author{
G. Schiwietz, P. Grande, ${ }^{(a)}$ B. Skogvall, J. P. Biersack, R. Köhrbrück, K. Sommer, A. Schmoldt, \\ P. Goppelt, I. Kádár, ${ }^{(b)}$ S. Ricz, ${ }^{(b)}$ and U. Stettner \\ Hahn-Meitner-Institut Berlin, Glienicker Strasse 100, D-1000 Berlin 39, Federal Republic of Germany
}

(Received 13 May 1991)

\begin{abstract}
The angle, energy, and fluence dependence of electron emission following the interaction of normally incident $100-\mathrm{MeV} \mathrm{Ne}^{9+}$ ions with thin polypropylene foils and $170-\mathrm{MeV} \mathrm{Ne}^{7+}$ projectiles with Mylar foils has been investigated experimentally. Spectra were taken for electron ejection angles of $0^{\circ}, 45^{\circ}$, and $120^{\circ}$ at fluences in the range of $2 \times 10^{13}$ to $4 \times 10^{15}$ ions $/ \mathrm{cm}^{2}$. A fluence-dependent carbon $K L L$ Auger energy shift of up to $68 \mathrm{eV}$ was observed. Model calculations for the nuclear track potential are consistent with the experimental findings.
\end{abstract}

PACS numbers: $61.80 . \mathrm{Jh}, 79.20 . \mathrm{Nc}, 79.60 . \mathrm{Eq}$

Nuclear track structures have been investigated for several decades. However, not much is known about the behavior of the solid during the first few femtoseconds after interaction with a fast heavy ion. Especially at energies of a few $\mathrm{MeV} / \mathrm{u}$, heavy ions produce a high degree of ionization and excitation in the solid. This may result in different types of defects along the projectile path. Investigations of conducting materials, especially of metallic glasses, have shown that the electronic energy loss of fast heavy ions may result in a plastic deformation of a solid [1]. For a more quantitative elucidation of this plastic deformation process or of the track formation in general [2], it is necessary to determine the ion-induced track potential, the corresponding electric field strength, and the lifetime of this field. In this work a first attempt is made to determine the track potential in the case of insulators. It will be shown that the nuclear track potential results in a considerable shift of target Auger electron energies.

We investigated the carbon $K$ Auger structure induced by normally incident $100-\mathrm{MeV} \mathrm{Ne} \mathrm{Ne}^{9+}$ ions on polypropylene (PP) and 170-MeV Ne ${ }^{7+}$ ions on Mylar foils. The experimental setup is similar to the one described in Ref. [3]. It consists mainly of an electrostatic tandem electron spectrometer, which may be moved around the target center in a magnetically shielded scattering chamber. All measurements were conducted at the VICKSI heavy-ion accelerator of the Hahn-Meitner-Institut Berlin $\mathrm{GmbH}$.

Thin target foils of $140 \pm 10 \mu \mathrm{g} / \mathrm{cm}^{2}$ were used, so that the incident ions only lose a small part of their initial energy in traversing the target. In order to avoid macroscopic charging of the foils or foil temperature in excess of 50 or $60^{\circ} \mathrm{C}$, about $20 \mu \mathrm{g} / \mathrm{cm}^{2}$ of $\mathrm{Al}$ was evaporated on the foils. This conducting film was placed on one side of a foil and spectra were taken for electrons ejected from the other side. In order to reduce the temperature load and to achieve a high accuracy for the determination of the ion fluence, a custom-made target wobbler was used in the $100-\mathrm{MeV} \mathrm{Ne}{ }^{9+}$ experiment. The motion of a target frame was synchronized with the energy scan of the electrostatic electron spectrometer. Thus, all points of an electron spectrum correspond to the same fluence and to the same number of incident ions. The ion current was varied between 0.3 and 2 particle $n A$.

Using two surface-barrier detectors to measure nuclear reaction products at angles of $28^{\circ}$ and $60^{\circ}$ with respect to the incident beam, the foil composition was monitored simultaneous to the measurement of the electron spectra. At a scattering angle of $28^{\circ}$ we observed high-energy reaction products below $70 \mathrm{MeV}$. These particles stem from quasielastic $\mathrm{Ne}+\mathrm{C}$ reactions, as was estimated with kinematic formulas. Thus, the number of fast reaction products was used to determine the carbon contents of the foils. Using C and PP targets of different thicknesses for calibration, we could determine that the particle spectrum at $60^{\circ}$ includes a $50 \%$ contribution from the $\mathrm{Ne}+\mathrm{H}$ reaction at energies below $20 \mathrm{MeV}$. This contribution is due to $(p, \alpha)$ and quasielastic reactions. Figure 1 displays the relative $\mathrm{C}$ and $\mathrm{H}$ contents of $\mathrm{PP}\left(\mathrm{C}_{3} \mathrm{H}_{6}\right)$ as a function of the fluence. It is seen that the number of $\mathrm{C}$ and $\mathrm{H}$ atoms per $\mathrm{cm}^{2}$ is continuously decreasing. Most likely this is due to a reduction of the foil thickness. The $\mathrm{H}$ content is decreasing much faster than the $\mathrm{C}$ content. This is known as carbonization and leads to an increased conductivity of the material [4]. The resistivity of polymer samples usually drops by several orders of magni-

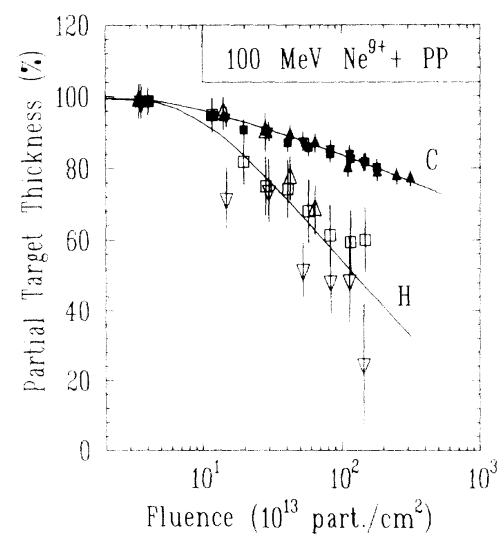

FIG. 1. Relative $\mathrm{H}$ and $\mathrm{C}$ contents of polypropylene samples irradiated with $100-\mathrm{MeV} \mathrm{Ne}^{9+}$ ions as a function of the fluence. The solid lines are drawn to guide the eye. 
tude. As known from previous investigations [5], the carbonization is accompanied by a blackening and a compaction of the target samples. The compaction could be observed in this work at fluences as low as $2 \times 10^{14}$ ions/ $\mathrm{cm}^{2}$. The color of the foils turned to light brown at fluences of about $10^{15}$ ions $/ \mathrm{cm}^{2}$ and they were nearly black at the highest fluences above $3 \times 10^{15}$ ions $/ \mathrm{cm}^{2}$. For even higher fluences the samples broke apart due to mechanical stress. At this point it should be emphasized that the chemical processes leading to the carbonization are of minor importance for the understanding of the short-time processes during the penetration of the solid by a fast heavy ion. From an auxiliary residual-gas analysis with a quadrupole mass spectrometer we determined the ratio of the losses of $\mathrm{H}$ to $\mathrm{C}$ atoms during the irradiation to be 6.7 in the case of $100-\mathrm{MeV} \mathrm{Ne}^{9+}$ projectiles. This is consistent with the data displayed in Fig. 1.

Figure 2 displays three electron spectra for the passage of $100-\mathrm{MeV} \mathrm{Ne}{ }^{9+}$ ions through a carbon foil and a polypropylene foil at two different fluences. The PP foil spectra for low fluences are dominated by the carbon $K$ Auger peak at $180 \mathrm{eV}$. This peak is shifted towards higher energies when the fluence is increased. In the case of carbon foils, the peak energy is similar to atomic Auger energies. The low-energy tail of the peak corresponds to emission of Auger electrons from up to $20 \AA$ below the surface. The deeper the electrons are produced inside the solid the more energy they lose on their way to the surface. In auxiliary measurements it was verified that the PP spectra depend on the observation angle and on the fluence, but not on the ion current. Thus, macroscopic charging of the sample does not take place. Consequently, the shift of the Auger peak can be assigned to the influence of a single track, in the case of low fluences.

Figure 3 displays the Auger energy shift for different ejection angles as a function of the fluence. The relative

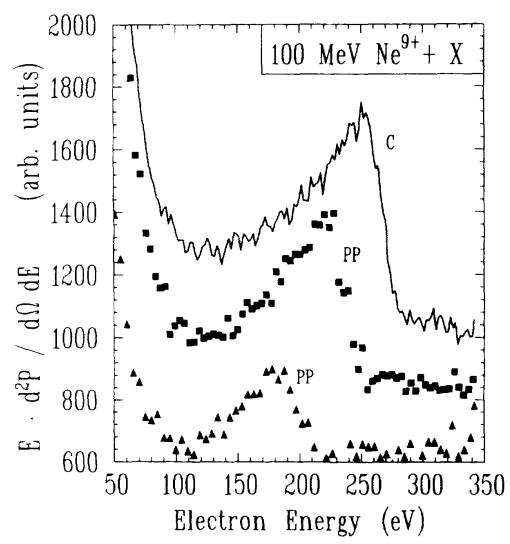

FIG. 2. Electron energy spectra taken at an ejection angle of $120^{\circ}$ for $100-\mathrm{MeV} \mathrm{Ne}{ }^{9+}$ ions on thin target foils. Solid line, $20-\mu \mathrm{g} / \mathrm{cm}^{2} \mathrm{C}$; squares, $1.55-\mu \mathrm{m}$ polypropylene at a fluence of $1.1 \times 10^{15}$ ions $/ \mathrm{cm}^{2}$; triangles, $1.55-\mu \mathrm{m}$ polypropylene at a fluence of $3.6 \times 10^{13}$ ions $/ \mathrm{cm}^{2}$. position of the PP $K$ Auger structure was determined by a fit to the corresponding carbon-foil spectra. Asymptotic energy shifts for zero fluence are $68 \pm 4 \mathrm{eV}$ at $120^{\circ}$ and $35 \pm 3 \mathrm{eV}$ at forward angles. The asymptotic value for $0^{\circ}$ Auger electron ejection in the $170-\mathrm{MeV}-\mathrm{Ne}^{7+}$ +Mylar experiment is $32 \pm 4 \mathrm{eV}$. It is seen from Fig. 3 that the energy shift tends to zero in the limit of high fluences. The lowest measured shift is $1 \pm 0.5 \mathrm{eV}$ at $3.1 \times 10^{15}$ ions $/ \mathrm{cm}^{2}$. At this fluence the ratio of $\mathrm{H}$ to $\mathrm{C}$ atoms is reduced by a factor of about 2.5 compared to $\mathrm{C}_{3} \mathrm{H}_{6}$, as may be extracted from Fig. 1. However, the chemical environment affects the carbon $K$ Auger peak energy by only about $5 \mathrm{eV}$ or less [6]. Therefore, we conclude that the transition from an insulator to a conducting material is responsible for the fluence dependence of the PP spectra.

In an insulator, recombination processes are strongly suppressed. Thus, for an inner-shell excited carbon atom inside a PP environment there is a high probability that its charge state is conserved during the decay time. The direct carbon $L$-shell ionization probability is about 0.48 [7] for a single electron in $100-\mathrm{MeV}-\mathrm{Ne}^{9+}+\mathrm{C}$ collisions. Also, taking into account that an Auger decay is only possible when there are at least two electrons left in the $L$ shell, the most probable $K$ Auger states in an insulator are $1 s 2 s^{2}, 1 s 2 s 2 p$, and $1 s 2 p^{2}$. The Auger energy for the transition from $1 s 2 s 2 p$ to $1 s^{2}$ to $22.6 \mathrm{eV}$ lower than the energy of the corresponding diagram lines. This was calculated with the atomic Hartree-Fock code by Cowan [8]. If the initial state is given by a four-electron configuration, the Auger energy is reduced by only about 10 $\mathrm{eV}$. In a conductor, recombination can take place before the Auger transition. This results in a population of the $1 s 2 s^{2} 2 p^{2}$ initial state and leads to an energy shift with

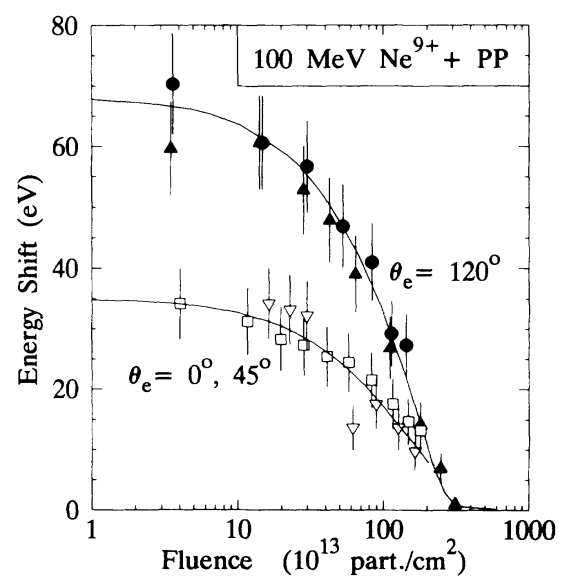

FIG. 3. Fluence dependence of the carbon $K$ Auger energy shift of $1.55-\mu \mathrm{m}$ polypropylene foils irradiated with $100-\mathrm{MeV}$ $\mathrm{Ne}^{9+}$ ions. The measurements were conducted at electron ejection angles of $0^{\circ}$ (open triangles), $45^{\circ}$ (open squares), and at $120^{\circ}$ (solid triangles and circles) for two target samples. The solid lines are drawn to guide the eye. 
respect to an insulator. In total one may estimate a shift of less then $25 \mathrm{eV}$ (the most probable energy shift is 18 eV) due to suppressed recombination and the presence of either hydrogen or carbon atoms near the Auger atom (chemical shift). The maximum observed shift is too large to be justified by the effects discussed above. Thus, the origin of the remaining shift of about $50 \mathrm{eV}$ must be explained.

In the following, it will be shown that this contribution of the Auger energy shift (about $50 \mathrm{eV}$ ) is due to the track potential. A highly charged ion produces a large amount of electronic vacancies on its way through the solid and any Auger electron which is ejected near the track will be decelerated according to the resulting track potential. In order to estimate the distribution of vacancies and trapped electrons inside the solid, transport theory has to be applied. Therefore, we extended the well-established Monte Carlo code TRIM [9], originally made for transport of ions in matter. The necessary modifications are as follows.

(i) The PP target is simulated by a statistical ensemble of screened carbon atoms ( 3 atoms per unit volume) and hydrogen atoms (6 atoms per unit volume). In addition twelve electrons with a binding energy of $11 \mathrm{eV}$, six electrons with a binding energy of $18 \mathrm{eV}$, and six electrons with a binding energy of $270 \mathrm{eV}$ are introduced in the target description. There are no explicit internal atomic energy losses in this model, since all particles may lose their energy via binary collisions with target electrons or atoms. Collective excitations, e.g., plasmon creation [10], are neglected in the model and are expected to increase the electronic stopping power by only $10 \%$ or less $[11,12]$.

(ii) Collisions between two electrons are described by Rutherford cross sections with a cutoff impact parameter, whereas all other cross sections are based on screened potentials. It was verified that the resulting track potential is rather insensitive of this cutoff.

(iii) In order to simulate the electronic target structure, excitation of inner-shell electrons into occupied bands is suppressed, simply by skipping the corresponding binary collision. This is necessary, since in general a classical collision theory incorporates neither quantized transition energies nor the Pauli exclusion principle.

The track potential $V_{T}(\mathbf{R})$ was computed from the spatial charge density of electronic vacancies $\rho_{v}(\mathbf{r})$ for all bands and from the charge density of stopped electrons $\rho_{s}(\mathbf{r})$ via

$V_{T}(\mathbf{R})=\int d \mathbf{r} \frac{\rho_{v}(\mathbf{r})-\rho_{s}(\mathbf{r})}{|\mathbf{R}-\mathbf{r}|}$, for $|\mathbf{R}-\mathbf{r}|>1.5 \AA$.

The maximum potential inside a PP foil of $10000 \AA$ is about $35 \mathrm{eV}$ according to the above described model. A closer examination of the numerical integral in Eq. (1) shows that the dominant part of the potential is of shortrange type with a typical range of $20 \AA$. This range is somewhat larger then the so-called infratrack, where the primary ion-solid interaction dominates the creation of defects. The radius of this cylinder is equal to the ion velocity in atomic units [13]. Thus, the radius of the infratrack is $7 \AA$ for the present case. The calculated track potential drops to about half its maximum value near both surfaces. Since carbon Auger electrons may escape the surface only from a depth of up to $20 \AA$, an average Auger energy shift of $20 \mathrm{eV}$ may be extracted from the results of the calculation.

A comparison of ionization cross sections for 100$\mathrm{MeV}-\mathrm{Ne}^{9+}+\mathrm{H}$ collisions calculated using the quantummechanical plane-wave Born approximation (PWBA) [14] for the three-body problem with binary-encounter (Rutherford) cross sections shows that the production of zero-energy electrons is underestimated in our Monte Carlo code by a factor of 5.2. In an earlier investigation we have shown that experimental electron energy spectra for $100-\mathrm{MeV}-\mathrm{Ne}^{10+}+\mathrm{He}$ collisions agree with PWBA cross sections to within $30 \%$ [15]. Since low-energy electrons are most important for the short-range potential, the classical Monte Carlo result for the track potential should be too low by a factor of 2 to 5 . An additional reduction of the potential of the order of $25 \%$ is expected, since our theoretical estimate does not incorporate either collective excitations in the electron energy loss $[10,16]$ or the interaction of directly ionized electrons with the track potential [17]. Based on this, the origin of the additional Auger energy shift of $50 \mathrm{eV}$ is consistent with a dominant contribution due to the track potential. Using the calculated range of $20 \AA$, the track potential is equivalent to an average radial electric field strength on the order of 5 $\mathrm{V} / \AA$ inside the solid.

From the above discussion it becomes clear that the measured maximum Auger energy shift is mainly due to the track potential and that the fluence dependence of the shift is correlated to recombination processes in the carbonized environment of a track. Since the solid is completely carbonized in the limit of high fluences, the PP Auger spectra become nearly identical to the corresponding carbon-foil spectra. In this limit recombination takes place within less than 100 a.u., i.e., before the Auger decay. Therefore the carbon $K$ Auger energies from solid carbon, irradiated PP (see Figs. 2 and 3 ) and singly ionized atomic carbon [8] are equal to within less than $5 \mathrm{eV}$. It is emphasized that the measured carbon-foil Auger energies for forward and backward angles are identical to within an experimental uncertainty of $\pm 0.5 \mathrm{eV}$. However, this is not the case for the PP spectra at low fluences.

As it was found recently [3], Auger electrons in the forward direction are partially produced [(20-50)\%] via electron-electron collision cascades. A significant part of these electrons should be created far away ( $\gg 20 \AA)$ from the track. Thus, they will not be affected by the track potential. In order to test the influence of electronic collision cascades on the results presented in Fig. 3, we fitted the sum of a shifted and an unshifted carbon-foil Auger spectrum to the PP spectra at forward angles. The results of this multiparameter fit indicate that only about 
$15 \%$ of the Auger electrons are created far away from the track and that the Auger energy shifts displayed in Fig. 3 are $3 \mathrm{eV}$ too low at forward angles and low fluences. Hence, the forward-backward asymmetry of the PP Auger energy shift is not due to electronic collision cascades. Furthermore, it is emphasized that the initial charge state $q_{i}=9$ is nearly equal to the mean final charge state $q_{f}=9.1$, as determined in an auxiliary charge-state analysis [18]. Thus, charge-state equilibration in the foil also cannot explain the forward-backward asymmetry.

However, with respect to the electron-hole recombination processes, which lead to a reduction of the measured energy shift, there are significant differences between the backward and forward surfaces.

(I) Recombination may occur along the track, since the corresponding volume is highly ionized and might give rise to high electron transport velocities. Typical $K$ Auger decay times are about 400 to 500 a.u. for highly ionized carbon, whereas the projectile needs 2000 a.u. to pass the solid. Thus, at the backward (ion-entrance) surface the projectile is still inside the solid, when an Auger decay occurs. However, at forward ejection the projectile may produce a conducting track between both surfaces before the Auger decay and recombination takes place via the aluminum grounding plane.

(II) Recombination may also occur within the local surrounding of an electronic vacancy, as in a conductor. At the backward surface, secondary electrons are produced simultaneous with the Auger states and most of the secondary electrons are ejected into forward directions. At the forward (ion-exit) surface, these electrons are already decelerated near the Auger atom by the time the Auger state is produced and thus recombination processes are faster. However, from the present data it is not possible to decide which process is dominant.

In summary, we have investigated the angle and fluence dependence of carbon $K L L$ Auger electron emission in collisions of fast highly charged $\mathrm{Ne}$ ions with thin polypropylene samples. In comparison to graphite foils, the measured carbon $K L L$ Auger energies from polypropylene are $68 \mathrm{eV}$ lower at backward angles and low fluences. Model calculations for the nuclear track potential are consistent with the experimentally observed maximum Auger energy shift. Thus, the measurement of Auger electrons in ion-solid interactions provides a sensitive tool to determine the track potential a few hundred atomic time units after the penetration of a surface. In the case of forward ejection or at fluences above $10^{14}$ ions $/ \mathrm{cm}^{2}$ the Auger energy shift is significantly reduced due to recombination processes. However, the details of these recombination processes are not fully understood and should be the subject of further studies.

We are much indebted to $M$. Briere and N. Stolterfoht for helpful comments on the present work. We thank W. Altmann and $G$. Chedor for their engaged cooperation in the construction of the target wobbler. P. Grande is an Alexander von Humboldt fellow.

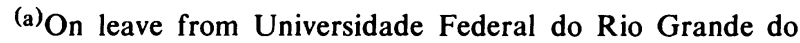
Sul, Rio Grande do Sul, Brazil.

(b) Permanent address: ATOMKI, Debrecen, Hungary.

[1] S. Klaumünzer, Ming-dong Hou, and G. Schumacher, Phys. Rev. Lett. 57, 850 (1986); S. Klaumünzer, Radiat. Eff. Defects Solids 110, 79 (1989).

[2] R. Spohr, Ion Tracks and Microtechnology (Vieweg, Braunschweig, 1990).

[3] G. Schiwietz, D. Schneider, J. P. Biersack, N. Stolterfoht, D. Fink, A. Mattis, B. Skogvall, H. Altervogt, V. Montemayor, and U. Stettner, Phys. Rev. Lett. 61, 1677 (1988).

[4] Proceedings of the Fourth International Conference on Radiation Effects in Insulators, Lyon, France, 1987, edited by P. Thevenard, A. Perez, J. Davenas, and H. Matzke [Nucl. Instrum. Methods Phys. Res., Sect. B 32 (1988)].

[5] S. L. Koul, I. D. Campbell, D. C. McDonald, L. T. Chadderton, D. Fink, J. P. Biersack, and M. Müller, Nucl. Instrum. Methods Phys. Res., Sect. B 32, 186 (1988).

[6] K. Siegbahn et al., Electron Spectroscopy for Chemical Analysis Applied to Free Molecules (North-Holland, Amsterdam, 1969).

[7] G. Schiwietz, Phys. Rev. A 42, 296 (1990).

[8] Total ionic energies (under neglect of configuration interaction) were calculated with the multiconfiguration Hartree-Fock code by R. D. Cowan, The Theory of Atomic Structure and Spectra (University of California, Berkeley, 1981).

[9] J. P. Biersack, Nucl. Instrum. Methods Phys. Res., Sect. B 19, 32 (1987).

[10] O. Sueoka, J. Phys. Soc. Jpn. 20, 2226 (1965).

[11] T. L. Ferrell and R. H. Ritchie, Phys. Rev. B 16, 115 (1977).

[12] I. Egri, Solid State Commun. 44, 563 (1982).

[13] W. Brandt and R. H. Ritchie, in Physical Mechanisms in Radiation Biology, edited by R. D. Cooper and R. W. Woods (Technical Information Center, Oakridge, TN, 1974), p. 20.

[14] D. R. Bates and G. Griffing, Proc. Phys. Soc. London A 66, 961 (1953).

[15] G. Schiwietz, B. Skogvall, N. Stolterfoht, D. Schneider, V. Montemayor, and H. Platten, Nucl. Instrum. Methods Phys. Res., Sect. B 40/41, 178 (1989); G. Schiwietz, H. Platten, D. Schneider, T. Schneider, W. Zeitz, K. Musiol, R. Kowallik, and N. Stolterfoht, Hahn-Meitner-Institut Report No. B-477, 1987 (unpublished), ISSN 0175-8349.

[16] A value of about $35 \mathrm{eV}$ for the maximum potential inside the bulk was obtained from a Monte Carlo calculation including classical collision cross sections as well as electron-phonon and electron-plasmon couplings [A. Akkerman (private communication)].

[17] This interaction will affect mainly low-energy electrons deep inside the solid. Fast electrons will experience no retardation at all, since electronic target screening reduces the time-dependent track potential.

[18] G. Schiwietz, Radiat. Eff. Defects Solids 112, 195 (1990). 